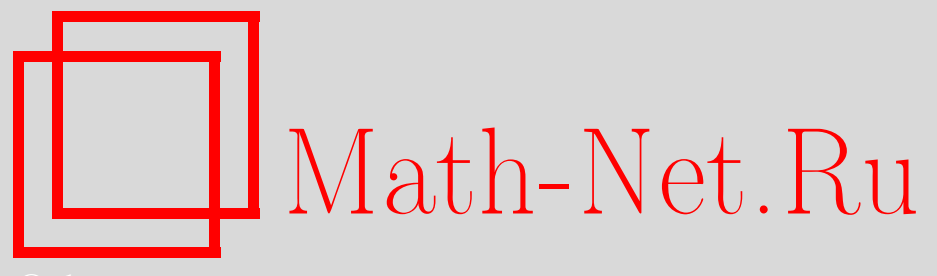

М. Ф. Раца, А. Г. Русу, О некоторых свойствах полных по выразимости систем формул в логике доказуемости Геделя-Леба, Дискрет. матем., 2000, том 12, выпуск 4, 63-82

DOI: https://doi.org/10.4213/dm354

Использование Общероссийского математического портала Math-Net.Ru подразумевает, что вы прочитали и согласны с пользовательским соглашением http://www.mathnet.ru/rus/agreement

Параметры загрузки:

IP : 54.224 .60 .19

26 апреля 2023 г., 10:01:17

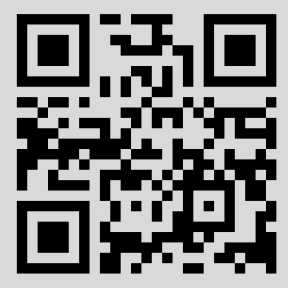


УдК 510

\section{О некоторых свойствах полных по выразимости систем формул в логике доказуемости Геделя-Леба}

(C) 2000 г. M. Ф. Раца, А. Г. Русу

Хорошо известны идеи о погружении интуиционистской логики в модальную логику с целью последующей интерпретации модальности доказуемо как выводимость в арифметике Пеано, а также возникающие при этом трудности. Р. М. Соловей и А. В. Кузнецов ввели в рассмотрение логику доказуемости Геделя-Леба, формулы которой построены из пропозициональных переменных с помощью связок $\&, \vee, \supset, \neg$ и $\Delta$ (геделизированная доказуемость). Логика эта определена классическим исчислением высказываний, обогащенным тремя $\Delta$-аксиомами

$$
\Delta(p \supset q) \supset(\Delta p \supset \Delta q), \quad \Delta(\Delta p \supset p) \supset \Delta p, \quad \Delta p \supset \Delta \Delta p,
$$

а также правилом усиления (правило Геделя). Формула называется (функционально) выразимой в логике $L$ через систему формул $\Sigma$, если ее можно получить из $\Sigma$ и переменных посредством ослабленного правила подстановки и правила замены эквивалентным в $L$. Понятия полноты и предполноты (по выразимости) в логике определяются традиционным образом. Система $\Sigma$ называется формульным базисом в логике $L$, если $\Sigma$ полна и независима в $L$.

В статье доказано, что в логике доказуемости Геделя-Леба и ряде ее расширений существует счетное семейство предполных классов формул, существуют формульные базисы любой конечной длины и отсутствует финитная аппроксимируемость по полноте.

\section{1. Введение}

В математической логике, алгебре, дискретной математике и их приложениях часто рассматривают отношение (функциональной) выразимости, означающее возможность получения одних операций (формул, функций) из других с помощью заранее фиксированных средств. Понятие выразимости (то есть представимости) одних булевых функций через другие посредством суперпозиций восходит к работам Э. Поста $[26,27]$. Большее продвижение было достигнуто в изучении важнейшего частного случая выразимости, а именно, свойства полноты по выразимости, когда рассматривается возможность получения всех операций данной логики. Упомянем, что А. В. Кузнецов [2] доказал существование критерия полноты систем функций в $k$-значной логике, С. В. Яблонский $[18,19]$ построил критерий полноты в полной 
3-значной логике в терминах 18 предполных классов функций, определяемых некоторыми предикатами [8], и описал ряд семейств таких классов в полной $k$-значной логике при $k>3$, а И. Розенберг [29] получил аналогичный критерий в произвольной полной $k$-значной логике. Предложив понятие итеративной алгебры Поста, А. И. Мальцев [9] заложил основы алгебраической интерпретации этих вопросов.

Естественно возни́кла задача исследования аналогичных вопросов в разнообразных логиках, определяемых исчислениями и необязательно обладающих адекватными конечными моделями. Среди последних упомянем интуиционистскую логику [1], модальные логики [15], логики доказуемости $[5,32]$, классическую логику предикатов первого порядка [17]. Основным объектом рассмотрения в них являются не функции, а формулы, определяемые синтаксически, без обязательного указания интерпретации. А. В. Кузнецов $[3,4]$ подходящим уточнением распространил понятие выразимости на случай формул. Полученные впоследствии решения проблем полноты по выразимости в интуиционистской логике высказываний [10], в модальной логике $S 5$ [12] и в классической логике предикатов первого порядка [6] основаны на конечных семействах предполных классов формул. Следовательно, мощности базисов (то есть полных и независимых систем формул) в этих логиках ограничены некоторыми фиксированными числами. Однако в модальной логике $S 4$ ситуация оказалась существенно иной ввиду обнаружения в ней базисов любой конечной длины [11], что приводит к невозможности существования в ней критерия полноты, основанного на конечном числе предполных классов формул.

В настоящей статье рассматривается логика доказуемости Геделя-Леба $[5,21$, $22,23,32]$ и обнаруживается, что с точки зрения вопросов полноты по выразимости эта логика ближе к модальной логике $S 4$ нежели, например, к модальной логике $S 5$ или к логике предикатов первого порядка. В частности, строится счетное семейство попарно различных предполных в ней классов формул. Затем для любого целого положительного числа $k$ дается пример базиса длины $k$.

О логике $L$ мы скажем, что она является финитно аппроксимируемой по (функциональной) полноте, если для любой неполной в ней системы формул существует табличное расширение логики $L$, в котором эта система также не полна. В работе доказывается и более сильный результат, а именно, что логика доказуемости Геделя-Леба не является даже финитно аппроксимируемой по полноте. Попутно эти явления распространяются также на все те расширения этой логики, которые не являются локально табличными (то есть локально конечными) логиками. В доказательствах используется алгебраическая интерпретация логик доказуемости, состоящая из диагонализируемых алгебр [24], именуемых также алгебрами Магари [5].

\section{2. Некоторые понятия и положения}

Формулы пропозициональной логики доказуемости $[5,32]$ определяем обычным образом на основе алфавита, состоящего из пропозициональных переменных $p, q, r, \ldots$, иногда с индексами, знаков операций \& (конъюнкции), $V$ (дизъюнкции), つ (импликации), ᄀ (отрицания) и $\Delta$ (геделизированной доказуемости), а также круглых скобок. Произвольные формулы обозначаем, как правило, заглавными латинскими буквами $A, B, C, \ldots$, возможно, с индексами. А формулы

$$
(p \& \neg p), \quad(p \supset p), \quad(\Delta \Delta p), \quad(p \& \Delta p), \quad((A \supset B) \&(B \supset A))
$$


обозначаем соответственно символами

$$
0, \quad 1, \quad \Delta^{2} p, \quad \square p, \quad A \sim B
$$

Внешние скобки формул часто будем опускать. Условимся писать $A\left(\pi_{1}, \ldots, \pi_{n}\right)$, если формула $A$ не содержит иных переменных кроме $\pi_{1}, \ldots, \pi_{n}$. Логической длиной формулы назовем число всех вхождений в нее логических операторов $\&, \vee, \supset, \neg$ и $\Delta$.

Как известно [5, 32], исчисление доказуемости Геделя-Леба, обозначаемое ниже символом $G L$, задается аксиомами классического исчисления высказываний, тремя $\Delta$-аксиомами

$$
\Delta p \supset \Delta^{2} p, \quad \Delta(p \supset q) \supset(\Delta p \supset \Delta q), \quad \Delta(\Delta p \supset p) \supset \Delta p
$$

а также тремя правилами вывода: правилом подстановки, правилом modus ponens, правилом усиления. Напомним, что последнее правило позволяет перейти от формулы $A$ к формуле $\Delta A$, а последняя формула из списка $\Delta$-аксиом называется аксиомой Леба $[5,23]$. Этому исчислению соответствует логика доказуемости Геделя-Леба, которую уточняем как семейство всех формул, выводимых в исчислении $G L$. Обозначим эту логику также символом $G L$. Для любой формулы $A$ запись $\vdash A$ будет означать, что $A$ выводима в исчислении $G L$ или, что то же самое, $A$ принадлежит логике $G L$ как множеству. Например, имеют место соотношения

$$
\begin{aligned}
\vdash \Delta(\Delta p \supset p) & \sim \Delta p, \\
\vdash \Delta(p \& q) & \sim(\Delta p \& \Delta q), \\
\vdash \square(p \& q) & \sim(\square p \& \square q) .
\end{aligned}
$$

Вообще, любое множество формул, содержащее все аксиомы исчисления $G L$ и замкнутое относительно трех ее правил вывода, называется (нормальной) логикой доказуемости [7]. Если одна из двух логик доказуемости включена в другую, то последнюю называют расширением предыдушей. Будем говорить, что формула $A$ верна в логике $L$, если $A$ принадлежит $L$ как множеству. Формулы $A$ и $B$ называются эквивалентными в $L$, если в $L$ верна их эквиваленция $(A \sim B)$. Семейство логик доказуемости упорядочено по включению и образует алгебраическую решетку. Важные свойства последней изучались В. Блоком [20], К. Сморинским [31], Л. Л. Максимовой [7], А. В. Чагровым [16] и др.

Для интерпретации формул будем использовать алгебраическую семантику логики $G L$ и ее расширений, которая состоит из диагонализируемых алгебр [24]. Эти алгебры имеют вид

$$
\mathfrak{A}=\langle E ; \Omega\rangle, \quad \Omega=\{\&, \vee, \supset, \neg, \Delta\}
$$

причем система $\langle E ; \&, \vee, \supset, \neg\rangle$ является булевой алгеброй, а унарная операция $\Delta$ удовлетворяет $\Delta$-тождествам

$$
\Delta(x \supset x)=(x \supset x), \quad \Delta(\Delta x \supset x)=\Delta x, \quad \Delta(x \& y)=(\Delta x \& \Delta y) .
$$

Операции сигнатуры алгебры обозначены теми же знаками, что и логические связки для удобства интерпретации формул на алгебрах, то есть сопоставления каждой

3 Дискретная математика, т.12 №4 
формуле той операции, которая выражается ею на множестве элементов этой алгебры.

Для любых элементов $\alpha, \beta \in E$ частичный порядок $\alpha \leqslant \beta$ определяется равенством $\alpha=(\alpha \& \beta)$, причем наименьший и соответственно наибольший элементы суть $0=(\alpha \& \neg \alpha)$ и $1=(\alpha \supset \alpha)$. Элемент $\alpha$ алгебры $\mathfrak{A}$ называется открытым, если $\square \alpha=\alpha$, то есть если $\alpha$ совпадает со своей внутренностью [28]. Ввиду очевидного равенства $\square \Delta \alpha=\Delta \alpha$ элемент $\Delta \alpha$ всегда открыт. Поэтому в качестве элемента $\Delta \alpha$ подходит дизъюнкция открытых атомов элемента $\square \alpha$. В любой диагонализируемой алгебре элементы 0 и 1 являются открытыми, поэтому любая невырожденная такая алгебра имеет по меньшей мере два открытых элемента.

Рассмотрим алгебру

$$
\mathfrak{M}=\langle M ; \Omega\rangle,
$$

носитель которой $M$ есть множество всех бесконечных последовательностей, имеющих вид

$$
\alpha=\left\langle\mu_{1}, \mu_{2}, \ldots\right\rangle
$$

и не содержащих иных членов, кроме 0 и 1 ; булевы операции над элементами из $M$ (то есть над бесконечными двоичными последовательностями) определяются покомпонентно как обычные булевы функции на множестве $\{0,1\}$, а $\Delta \alpha$ есть такая последовательность

$$
\left\langle 1, \nu_{1}, \nu_{2}, \ldots\right\rangle
$$

что

$$
\nu_{i}=\left(\mu_{1} \& \ldots \& \mu_{i}\right)
$$

при всяком $i=1,2, \ldots$ (cp. [25]). Легко проверить, что алгебра $\mathfrak{M}$ является диагонализируемой. Ноль и единица новой алгебры суть элементы $\langle 0,0, \ldots\rangle$ и $\langle 1,1, \ldots\rangle$ соответственно.

Под оценкой $\varphi$ переменных формулы $A\left(p_{1}, \ldots, p_{n}\right)$ будем понимать отображение $\varphi$ множества ее переменных в множество элементов рассматриваемой алгебры. При этом условимся писать

$$
A\left(\varphi\left(p_{1}\right), \ldots, \varphi\left(p_{n}\right)\right)=\varphi(A) .
$$

Будем говорить, что формула верна на данной диагонализируемой алгебре $\mathfrak{A}$, если при любой оценке переменных элементами из $\mathfrak{A}$ она равна единице этой алгебры. Будем говорить, что оценка $\varphi$ опровергает формулу $A$ на данной алгебре, если $\varphi(A)$ отлична от единицы этой алгебры. Известно [5], что для всякой диагонализируемой алгебры $\mathfrak{A}$ множество всех формул, верных на $\mathfrak{A}$, составляет некоторую логику доказуемости. Назовем ее логикой алгебры $\mathfrak{A}$ и обозначим через $L \mathfrak{A}$.

Логика называется табличной, если она совпадает с логикой некоторой конечной алгебры. Логика $L$ называется предтабличной, если она не таблична, но всякое ее собственное расширение уже является табличной логикой. Например, логика LM является предтабличной логикой доказуемости [7]. Логика доказуемости $L$ называется локально табличной, если всякая конечно-порожденная диагонализируемая алгебра, на которой верны все формулы из $L$, является конечной. Заметим, что логика $L \mathfrak{M}$, и тем более логика $G L$, не являются локально табличными, поскольку формулы $p, \Delta p, \Delta^{2} p, \ldots$ составляют бесконечную последовательность попарно не эквивалентных в $L \mathfrak{M}$, и тем более в $G L$, формул от одной и той же переменной $p$. Из результатов В. Блока [20], Л. Л. Максимовой [7] и А. В. Чагрова [16] вытекает следующее утверждение. 
Предложение 1. Любая логика доказуемости является локалъно табличной тогда и только тогда, когда она не включена в логику $L \mathfrak{M}$.

Будем говорить, что формула $A$ (функционально) выразима в логике $L$ через систему (множество, класс) формул $\Sigma$, если можно получить $A$, исходя из переменных и формул, принадлежащих $\Sigma$, посредством конечного числа применений ослабленного правила подстановки, позволяющего перейти от двух формул к результату подстановки одной из них в другую вместо всех вхождений одной и той же переменной, и правила замены эквивалентным в логике $L$, позволяющего перейти от одной формулы к любой эквивалентной ей в $L$ формуле. Система (формул) $\Sigma$ называется полной по выразимости в логике $L$, если все формулы языка этой логики выразимы в $L$ через $\Sigma$. Очевидно, что если какая-то система формул полна в логике $L$, то она полна и во всяком расширении $L$. Формула $A$ называется шефферовой в логике $L$, если система $\{A\}$ полна в $L$. Система (формул) $\Sigma$ называется предполной в логике $L$, если $\Sigma$ не полна в $L$, но для всякой формулы $A$, не выразимой в $L$ через $\Sigma$, система $\Sigma \cup\{A\}$ уже полна в $L$.

Символом $A\left[\pi_{1} / B_{1}, \ldots, \pi_{n} / B_{n}\right]$ (или, короче, $A\left[B_{1}, \ldots, B_{n}\right]$ ) обозначим результат подстановки в $A$ формул $B_{1}, \ldots, B_{n}$ вместо переменных $\pi_{1}, \ldots, \pi_{n}$ соответственно (вхождение в $A$ всех этих переменных не обязательно). Аналогично будем обозначать результат подстановки значений формул при интерпретациях. Будем говорить, что формула $A$, не содержащая иных переменных, кроме каких-то $\pi_{1}, \ldots, \pi_{n}$, сохраняет на алгебре $\mathfrak{A}$ предикат $R\left(x_{1}, \ldots, x_{m}\right)$, если для всевозможных элементов

$$
\beta_{i j}, \quad i=1, \ldots, m, \quad j=1, \ldots, n,
$$

алгебры $\mathfrak{A}$ из того, что истинны высказывания

$$
R\left(\beta_{11}, \beta_{21}, \ldots, \beta_{m 1}\right), \ldots, R\left(\beta_{1 n}, \beta_{2 n}, \ldots, \beta_{m n}\right),
$$

следует, что истинно также

$$
R\left(A\left[\pi_{1} / \beta_{11}, \pi_{2} / \beta_{12}, \ldots, \pi_{n} / \beta_{1 n}\right], \ldots, A\left[\pi_{1} / \beta_{m 1}, \pi_{2} / \beta_{m 2}, \ldots, \pi_{n} / \beta_{m n}\right]\right) .
$$

Очевидно, что класс всех формул, которые сохраняют на данной алгебре данный предикат, замкнут относительно выразимости в логике этой алгебры. Поэтому, если все формулы какой-то системы $\Sigma$ сохраняют на диагонализируемой алгебре $\mathfrak{A}$ некоторый предикат, а формула $A$ не сохраняет этого предиката, то $A$ невыразима через $\Sigma$ в логике $L \mathfrak{A}$. В таком случае скажем, что $A$ от $\Sigma$ отделяется на $\mathfrak{A}$ этим предикатом.

\section{3. Пример счетного семейства предполных классов формул}

Хорошо известна роль предполных классов для получения критериев полноты (по выразимости) в табличных логиках. Для случая логик, задаваемых исчислениями, эти классы также оказались полезными. Так, полученные ранее критерии полноты в интуиционистской логике высказываний [10] и в классической логике предикатов первого порядка [6] основаны соответственно на 12 и 6 предполных классах формул. В модальной логике $S 4$ был обнаружен факт существования бесконечного числа 
предполных классов [11], который не только говорит о возникновении принципиальных трудностей в поиске подходящего критерия полноты, но также ставит проблему существования в принципе такого критерия.

Покажем, что в логике доказуемости $G L$ и некоторых ее расширениях также можно построить пример бесконечного числа предполных классов. Для этого приведем ряд необходимых понятий и рассуждений.

Алгеброй формул называют алгебру вида (3), в которой носитель $E$ есть множество всех формул языка логики $G L$, а результатами применения ее операций к произвольным формулам $A$ и $B$ являются формулы $A \& B, A \vee B, A \supset B, \neg A$ и $\Delta A$ соответственно. Эта алгебра не является диагонализируемой, но становится таковой при рассмотрении формул с точностью до эквивалентности в рассматриваемой логике доказуемости $L$, то есть можно констатировать фактор-алгебру [28] алгебры формул по отношению эквивалентности в $L$. Полученная при этом диагонализируемая алгебра является линденбаумовой алгеброй (или алгеброй Линденбаума [28]) логики $L$. Логика этой алгебры совпадает с $L$. Формульной реализацией $[11,13]$ диагонализируемой алгебры $\mathfrak{A}$ в данной логике доказуемости $L$ будем называть такое отображение алгебры $\mathfrak{A}$ в множество формул, которое при рассмотрении формул с точностью до эквивалентности в $L$ становится изоморфизмом между алгеброй $\mathfrak{A}$ и некоторой подалгеброй линденбаумовой алгебры логики $L$.

Пусть $\mathfrak{M}^{*}$ - подалгебра алгебры $\mathfrak{M}$, порожденная нулевым элементом

$$
\langle 0,0, \ldots\rangle
$$

последней. Ясно, что открытые элементы обеих алгебр $\mathfrak{M}$ и $\mathfrak{M}^{*}$ суть одни и те же двоичные последовательности

$$
\begin{gathered}
\alpha_{0}=\langle 0,0,0, \ldots\rangle, \quad \alpha_{1}=\langle 1,0,0, \ldots\rangle, \\
\alpha_{2}=\langle 1,1,0,0, \ldots\rangle, \ldots, \alpha_{\omega}=\langle 1,1,1, \ldots\rangle
\end{gathered}
$$

и только они. Открытые элементы образуют бесконечную цепь

$$
\alpha_{1}<\alpha_{2}<\ldots<\alpha_{i}<\ldots
$$

Очевидно, что $\alpha_{1}=\Delta \alpha_{0}$, а при любом целом положительном $i$ имеет место равенство $\alpha_{i}=\Delta^{i} \alpha_{0}$. О бесконечной двоичной последовательности

$$
\left\langle\mu_{1}, \mu_{2}, \ldots\right\rangle
$$

скажем, что она стабилизирована, если существует целое положительное $s$, что

$$
\mu_{s}=\mu_{s+1}=\ldots
$$

Лемма 1. Стабилизированные бесконечные двоичные последовательности и только они являются элементами алгебры $\mathfrak{M}^{*}$.

Дохазательство. Действительно, применение сигнатурных операций алгебры $\mathfrak{M}^{*}$ над любыми стабилизированными последовательностями всегда дает такие же последовательности. Из этого факта и того, что алгебра $\mathfrak{M}^{*}$ порождается нулевым, заведомо стабилизированным элементом, следует, что любой элемент этой алгебры 
является стабилизированной последовательностью. Обратно, пусть последовательность

$$
\alpha=\left\langle\mu_{1}, \mu_{2}, \ldots\right\rangle
$$

является стабилизированной. Тогда существует такое целое положительное $s$, что $\mu_{s}=\mu_{s+1}, \ldots$ Индукцией по $s$ докажем, что $\alpha$ является элементом алгебры $\mathfrak{M}^{*}$. Если $s=1$, то $\alpha$ либо совпадает с нулем $0=\langle 0,0, \ldots\rangle$, либо является отрицанием этого элемента и тем самым совпадает с единицей алгебры $\mathfrak{M}^{*}$. Пусть $s>1$ и пусть по индуктивному предположению все последовательности вида

$$
\beta=\left\langle\nu_{1}, \nu_{2}, \ldots\right\rangle
$$

где $\nu_{s-1}=\nu_{s}=\ldots$, являются элементами алгебры $\mathfrak{M}^{*}$. В случае, когда $\mu_{s-1}=\mu_{s}$, элемент $\alpha$ является последовательностью вида $\beta$ и поэтому принадлежит алгебре $\mathfrak{M}^{*}$. Пусть $\mu_{s-1} \neq \mu_{s}$. Тогда зафиксируем элемент алгебры $\mathfrak{M}^{*}$

$$
\beta=\left\langle\mu_{1}, \ldots, \mu_{s-2}, \mu_{s-1}, \mu_{s-1}, \ldots\right\rangle \text {. }
$$

Возможны два случая, $\mu_{s-1}=0, \mu_{s}=1$ и $\mu_{s-1}=1, \mu_{s}=0$. В первом случае $\alpha=\Delta^{s-1} 0 \supset \beta$ и поэтому $\alpha \in \mathfrak{M}^{*}$. Во втором случае равенство $\alpha=\beta \& \Delta^{s-1} 0$ также подтверждает, что $\alpha \in \mathfrak{M}^{*}$. Лемма доказана.

Следующая лемма показывает, что логика $L \mathfrak{M}$ является устойчивой относительно замены алгебры $\mathfrak{M}$ на более простую ее подалгебру $\mathfrak{M}^{*}$.

Лемма 2. Справедливо равенство $L \mathfrak{M}=L \mathfrak{M}^{*}$.

Доказательство. Действительно, включение $L \mathfrak{M} \subseteq \mathfrak{M}^{*}$ вытекает из того, что $\mathfrak{M}^{*}$ есть подалгебра алгебры $\mathfrak{M}$. Обратно, пусть формула $A\left(p_{1}, \ldots, p_{n}\right)$ принадлежит логике $L \mathfrak{M}^{*}$. Предположим, что $A \notin L \mathfrak{M}$. Тогда существует оценка $\varphi$ переменных формулы элементами алгебры $\mathfrak{M}$, опровергающая на ней эту формулу и, тем самым, имеет место соотношение

$$
\varphi(A)=\left\langle\nu_{1}, \nu_{2}, \ldots\right\rangle \neq 1 .
$$

Следовательно, существует целое положительное $j$ такое, что $\nu_{j}=0$. Исходя из этого, далее построим новую оценку $\psi$ переменных формулы $A$ элементами подалгебры $\mathfrak{M}^{*}$, также опровергающую $A$, но уже на $\mathfrak{M}^{*}$. Пусть оценка $\varphi$ произвольной переменной $p_{i}$ задается равенством

$$
\varphi\left(p_{i}\right)=\left\langle\mu_{1}, \ldots, \mu_{j}, \mu_{j+1}, \ldots\right\rangle .
$$

Тогда оценку $\psi$ определяем равенством

$$
\psi\left(p_{i}\right)=\left\langle\mu_{1}, \ldots, \mu_{j}, \mu_{j}, \ldots\right\rangle .
$$

Другими словами, $\psi\left(p_{i}\right)$ есть последовательность, в которой первые $j$ членов совпадают с первыми $j$ членами последовательности $\varphi\left(p_{i}\right)$, а все последующие члены равны $\mu_{j}$. На основании леммы 1 заключаем, что последовательность $\psi\left(p_{i}\right)$ является элементом алгебры $\mathfrak{M}^{*}$. Поскольку $p_{i}$ является произвольной среди переменных $p_{1}, \ldots, p_{n}$, а индекс $j$ является общим при оценке $\varphi$, индукцией по логической длине формулы $A$ легко убедиться, что верно равенство

$$
\psi(A)=\left\langle\nu_{1}, \ldots, \nu_{j-1}, 0,0, \ldots\right\rangle
$$


которое опровергает допущение, что $A \in L \mathfrak{M}^{*}$. Следовательно, формула $A$ принадлежит логике $L \mathfrak{M}$.

Лемма доказана.

По лемме 1 каждый элемент алгебры $\mathfrak{M}^{*}$ является стабилизированной двоичной последовательностью. Заметим, что для любого открытого элемента $\alpha_{i}, i=1,2, \ldots$, импликация $\alpha_{i+1} \supset \alpha_{i}$ есть последовательность, содержащая 0 на $(i+1)$-м месте и 1 на всех остальных местах. Поэтому для каждого элемента $\beta$ алгебры $\mathfrak{M}^{*}$ существует его каноническая форма $[11,13]$, которая может быть двух видов:

$$
\left(\alpha_{i+1} \supset \alpha_{i}\right) \&\left(\alpha_{j+1} \supset \alpha_{j}\right) \& \ldots \&\left(\alpha_{k+1} \supset \alpha_{k}\right), \quad i<j<k,
$$

когда последовательность $\beta$ содержит 0 на местах с номерами $i+1, j+1, \ldots, k+1$ и содержит бесконечное число раз 1 на всех остальных местах, и

$$
\left(\alpha_{i+1} \supset \alpha_{i}\right) \&\left(\alpha_{j+1} \supset \alpha_{j}\right) \& \ldots \&\left(\alpha_{k+1} \supset \alpha_{k}\right) \& \alpha_{l}, \quad i<j<k<l,
$$

когда последовательность $\beta$ содержит 0 на местах с номерами

$$
i+1, j+1, \ldots, k+1, l+1, l+2, \ldots
$$

и содержит 1 конечное число раз на всех остальных местах. Каноническая форма любого элемента алгебры $\mathfrak{M}^{*}$ единственна с точностью до ассоциативности конъюнкции. Единица алгебры есть вырожденная конъюнкция, а именно, пустая.

Далее построим формульную реализацию алгебры $\mathfrak{M}^{*}$ в логике $G L$. Для этого рассмотрим формулы ряда

$$
0, \quad \Delta 0, \quad \Delta^{2} 0, \ldots, 1,
$$

обозначив их символами $C_{0}, C_{1}, C_{2}, \ldots, 1$ соответственно. Заметим, что ввиду аксиомы $\Delta p \supset \Delta^{2} p$ и транзитивности импликации имеет место выводимость

$$
\vdash\left(C_{i} \supset C_{j}\right), \quad 0 \leqslant i \leqslant j .
$$

Кроме того, поскольку истинно высказывание

$$
\vdash\left(C_{i+1} \sim \Delta C_{i}\right), \quad i=0,1, \ldots,
$$

в силу соотношения (1) получаем, что

$$
\vdash\left(\Delta\left(C_{i+1} \supset C_{i}\right) \sim \Delta C_{i}\right), \quad i=0,1, \ldots .
$$

Каждому элементу $\beta$ алгебры $\mathfrak{M}^{*}$, имеющему каноническую форму (4) или (5), ставим в соответствие формулу

$$
\left(C_{i+1} \supset C_{i}\right) \&\left(C_{j+1} \supset C_{j}\right) \& \ldots \&\left(C_{k+1} \supset C_{k}\right)
$$

или соответственно формулу

$$
\left(C_{i+1} \supset C_{i}\right) \&\left(C_{j+1} \supset C_{j}\right) \& \ldots \&\left(C_{k+1} \supset C_{k}\right) \& C_{l},
$$

$i<j<\ldots<k<l$, которые являются результатами замены в (4) и (5) всех открытых элементов $\alpha_{0}, \alpha_{1}, \ldots$ на формулы $C_{0}, C_{1}, \ldots$ соответственно. Единице алгебры $\mathfrak{M}^{*}$ (имеющей каноническую форму в виде пустой конъюнкции) ставим в соответствие формулу 1. Тем самым получаем инъективное отображение алгебры $\mathfrak{M}^{*}$ в множество формул. Отображение это обозначаем буквой $f$, при этом образ элемента $\beta$ записываем символом $f(\beta)$. 
Лемма 3. Для любых элементов $\beta$ и $\gamma$ алгебры $\mathfrak{M}^{*}$ справедливы следующие три выводимости:

$$
\begin{aligned}
\vdash(f(\beta \& \gamma) & \sim(f(\beta) \& f(\gamma))), \\
\vdash(f(\Delta \beta) & \sim \Delta f(\beta)), \\
\vdash(f(\neg \beta) & \sim \neg f(\beta)) .
\end{aligned}
$$

Доказательство. Если $\beta=1$ или $\gamma=1$, то утверждение (11) очевидно. Пусть $\beta \neq 1$ и $\gamma \neq 1$. Тогда в канонической форме элемента $(\beta \& \gamma)$ встречается импликация $\left(\alpha_{r+1} \supset \alpha_{r}\right)$ (или $\alpha_{l}$ ) тогда и только тогда, когда она (соответственно $\alpha_{l}$ ) встречается в одноименной форме по крайней мере одного из элементов $\beta$ или $\gamma$. Из этого и определения отображения $f$ следует, что соответствующая импликация $C_{r+1} \supset C_{r}$ (или $C_{l}$ ) встречается в виде конъюнктивного члена в формуле $f(\beta \& \gamma)$ тогда и только тогда, когда она встречается аналогичным образом хотя бы в одной из формул $f(\beta)$ или $f(\gamma)$. Тем самым эквивалентность (11) доказана.

Докажем выводимость (12). Если $\beta=1$, то она справедлива. Пусть $\beta \neq 1$. Тогда $\beta$ имеет каноническую форму вида (4) или (5). Поэтому в силу перестановочности операции $\Delta$ с конъюнкцией \& получаем то или иное из равенств

$$
\Delta \beta=\Delta\left(\alpha_{i+1} \supset \alpha_{i}\right) \& \Delta\left(\alpha_{j+1} \supset \alpha_{j}\right) \& \ldots \& \Delta\left(\alpha_{k+1} \supset \alpha_{k}\right)
$$

или

$$
\Delta \beta=\Delta\left(\alpha_{i+1} \supset \alpha_{i}\right) \& \Delta\left(\alpha_{j+1} \supset \alpha_{j}\right) \& \ldots \& \Delta\left(\alpha_{k+1} \supset \alpha_{k}\right) \& \Delta \alpha_{l},
$$

где $i<j<\ldots<k<l$. Используя очевидное равенство

$$
\Delta\left(\alpha_{r+1} \supset \alpha_{r}\right)=\Delta \alpha_{r}
$$

при $r=1,2, \ldots$ и сравнимость открытых элементов, упрощаем предыдущие равенства и в результате получаем, что

$$
\Delta \beta=\Delta \alpha_{i}
$$

Поэтому имеет место соотношение

$$
\vdash\left(f(\Delta \beta) \sim f\left(\Delta \alpha_{i}\right)\right) .
$$

В силу определения открытых элементов

$$
\Delta \alpha_{i}=\alpha_{i+1}
$$

Поэтому

$$
\vdash\left(f\left(\Delta \alpha_{i}\right) \sim f\left(\alpha_{i+1}\right)\right) .
$$

Из определения отображения $f$ следует, что

$$
\vdash\left(f\left(\alpha_{i+1}\right) \sim C_{i+1}\right) .
$$

На основании выводимостей (14)-(16) и транзитивности отношения эквивалентности находим, что

$$
\vdash\left(f(\Delta \beta) \sim \Delta C_{i}\right)
$$


Формула $f(\beta)$ по определению отображения $f$ есть либо формула (9), либо (10). Значит, $\Delta f(\beta)$ есть соответственно одна из двух формул

$$
\Delta\left(\left(C_{i+1} \supset C_{i}\right) \&\left(C_{j+1} \supset C_{j}\right) \& \ldots \&\left(C_{k+1} \supset C_{k}\right)\right)
$$

или

$$
\Delta\left(\left(C_{i+1} \supset C_{i}\right) \&\left(C_{j+1} \supset C_{j}\right) \& \ldots \&\left(C_{k+1} \supset C_{k}\right) \& C_{l}\right) .
$$

Ввиду (2) оператор $\Delta$ перестановочен с конъюнкцией $\&$, поэтому $\Delta f(\beta)$ эквивалентна в логике $G L$ той или иной из формул

$$
\Delta\left(\left(C_{i+1} \supset C_{i}\right) \& \Delta\left(C_{j+1} \supset C_{j}\right) \& \ldots \& \Delta\left(C_{k+1} \supset C_{k}\right)\right)
$$

или

$$
\Delta\left(\left(C_{i+1} \supset C_{i}\right) \& \Delta\left(C_{j+1} \supset C_{j}\right) \& \ldots \& \Delta\left(C_{k+1} \supset C_{k}\right) \& C_{l}\right),
$$

где $i<j<\ldots<k<l$. Упрощая эти формулы с применением соотношений (5), (6) и (7), получаем, что

$$
\vdash\left(\Delta f(\beta) \sim \Delta C_{i}\right) .
$$

А из этого соотношения и (17) следует требуемое высказывание (12).

Наконец, докажем выводимость (13). С одной стороны, отправляемся от факта, что элемент $\beta$ имеет каноническую форму вида (4) или вида (5). Пусть $\beta$ имеет форму (4). Тогда элемент $\neg \beta$ есть такая двоичная последовательность, в которой на местах с номерами $i+1, j+1, \ldots, k+1$ стоят единицы, а на остальных нули. Поэтому элемент $\neg \beta$ имеет такую каноническую форму, что образ его $f(\neg \beta)$ при отображении $f$ есть формула

$$
\begin{array}{r}
\left(C_{1} \supset C_{0}\right) \& \ldots \&\left(C_{i} \supset C_{i-1}\right) \&\left(C_{i+2} \supset C_{i+1}\right) \& \ldots \&\left(C_{j} \supset C_{j-1}\right) \\
\&\left(C_{j+2} \supset C_{j+1}\right) \& \ldots \&\left(C_{k} \supset C_{k-1}\right) \& C_{k+1} .
\end{array}
$$

С другой стороны, поскольку $f(\beta)$ есть формула (9), имеет место выводимость

$$
\vdash\left(\neg f(\beta) \sim\left(\left(C_{i+1} \& \neg C_{i}\right) \vee\left(C_{j+1} \& \neg C_{j}\right) \vee \ldots \vee\left(C_{k+1} \& \neg C_{k}\right)\right)\right) .
$$

Заметим, что каждый дизъюнктивный член правой части этой эквиваленции влечет каждый конъюнктивный член формулы (18). Поэтому в рассматриваемом случае получаем, что

$$
\vdash(\neg f(\beta) \supset f(\neg \beta)) .
$$

Пусть теперь элемент $\beta$ имеет каноническую форму (5). Тогда элемент $\neg \beta$ есть такая двоичная последовательность, в которой на местах с номерами

$$
i+1, j+1, \ldots, k+1, l+1, l+2, \ldots
$$

стоят единицы, а на остальных нули. Следовательно, элемент $\neg \beta$ обладает такой канонической формой, что его образ $f(\neg \beta)$ при отображении $f$ есть формула

$$
\begin{aligned}
\left(C_{1} \supset C_{0}\right) \& \ldots & \&\left(C_{i} \supset C_{i-1}\right) \&\left(C_{i+2} \supset C_{i+1}\right) \& \ldots \&\left(C_{j} \supset C_{j-1}\right) \\
& \&\left(C_{j+2} \supset C_{j+1}\right) \& \ldots \&\left(C_{k} \supset C_{k-1}\right) \\
& \&\left(C_{k+2} \supset C_{k+1}\right) \& \ldots \&\left(C_{l} \supset C_{l-1}\right)
\end{aligned}
$$


в которой некоторые указанные в ней импликации могут быть лишними, если не выполняются дополнительные условия на индексы

$$
2 \leqslant i, \quad i+2<j, \quad j+2<k, \quad k+2<l .
$$

С другой стороны, поскольку $f(\beta)$ есть формула (10), имеет место эквиваленция

$$
\vdash\left(\neg f(\beta) \sim\left(\left(C_{i+1} \& \neg C_{i}\right) \vee\left(C_{j+1} \& \neg C_{j}\right) \vee \ldots \vee\left(C_{k+1} \& \neg C_{k}\right) \vee \neg C_{l}\right)\right) .
$$

Нетрудно проверить, что каждый дизъюнктивный член правой части этой эквиваленции влечет каждый конъюнктивный член формулы (20). Поэтому в рассматриваемом случае опять имеет место (19). Далее исходим из очевидного факта

$$
\vdash \neg f(\beta \& \neg \beta) .
$$

Ввиду доказанной выводимости (11) отсюда следует, что

$$
\vdash \neg(f(\beta) \& f(\neg \beta)) .
$$

А последнее эквивалентно соотношению

$$
\vdash(f(\neg \beta \supset \neg f(\beta)),
$$

которое вместе с (19) доказывает утверждение (13). Лемма доказана.

Следующая лемма является обобщением леммы 3 и показывает, что отображение $f$ является формульной реализацией алгебры $\mathfrak{M}^{*}$ в логике доказуемости ГеделяЛеба.

Лемма 4. Для любой формуль $F\left(p_{1}, \ldots, p_{n}\right)$ логики доказуемости и любъх элементов $\beta_{1}, \ldots, \beta_{n}$ алгебры $\mathfrak{M}^{*}$ справедливо общее утверждение

$$
\vdash f\left(F\left[p_{1} / \beta_{1}, \ldots, p_{n} / \beta_{n}\right]\right) \sim F\left[p_{1} / f\left(\beta_{1}\right), \ldots, p_{n} / f\left(\beta_{n}\right)\right] .
$$

В самом деле, утверждение легко доказывается индукцией по построению формулы $B$ с помощью соотношений (11) - (13), доказанных в лемме 3.

На основании леммы 4 заключаем, что при рассмотрении формул с точностью до эквивалентности в логике $G L$ отображение $f$ является изоморфизмом между алгеброй $\mathfrak{M}^{*}$ и некоторой подалгеброй линденбаумовой алгебры логики $G L$. Это означает, что $f$ является формульной реализацией алгебры $\mathfrak{M}^{*}$ в логике $G L$. Из определения этой формульной реализации видим, что элементам алгебры $\mathfrak{M}^{*}$ она ставит в соответствие лишь одноместные формулы. Множество этих формул с точностью до эквивалентности в логике $G L$, как и множество элементов алгебры $\mathfrak{M}^{*}$, является счетным.

Следующая лемма раскрывает одно свойство формульной реализации $f$, которое будет использовано ниже.

Лемма 5. При формульной реализации $f$ алгебры $\mathfrak{M}^{*}$ в логике GL любому элементу $\beta$ этой алгебры ставится в соответствие такая одноместная формула $f(\beta)$, что для любого элемента $\gamma$ этой же алгебры имеет место равенство

$$
f(\beta)[\gamma]=\beta
$$


Таблица 1. Пример счетного семейства предполных классов

\begin{tabular}{|l|l|l|l|}
\hline Класс & $\begin{array}{l}\text { Определяющий } \\
\text { предикат }\end{array}$ & \multicolumn{1}{|c|}{ Примеры формул, } \\
принадлежащих классу & \begin{tabular}{l} 
не принадлежащих классу \\
\hline$K_{1}$
\end{tabular} \\
\hline$x \leqslant \Delta 0$ & $\begin{array}{l}0, \Delta 0, \Delta^{2} 0 \supset p, \quad \Delta^{3} 0 \supset p, \\
\ldots, p \& q\end{array}$ & $\begin{array}{l}1, \Delta^{2} 0, \Delta^{3} 0, \ldots, \Delta 0 \supset p, \neg p, \Delta p, \\
p \vee q, p \supset q\end{array}$ \\
\hline$K_{2}$ & $\square x \leqslant \Delta^{2} 0$ & $\begin{array}{l}0, \Delta 0, \Delta^{2} 0, \Delta^{3} 0 \supset p, \Delta^{4} 0 \supset \\
p, \ldots, p \& q\end{array}$ & $\begin{array}{l}1, \Delta^{3} 0, \Delta^{4} 0, \ldots, \Delta 0 \supset p, \Delta^{2} 0 \supset \\
p, \neg p, \Delta p, p \vee q, p \supset q\end{array}$ \\
\hline$\ldots$ & $\ldots$ & $\ldots$ & $\ldots$ \\
\hline$K_{i}$ & $\square x \leqslant \Delta^{i} 0$ & $\begin{array}{l}0, \Delta 0, \Delta^{2} 0, \ldots, \Delta^{i} 0, \Delta^{i+1} 0 \supset p, \\
\Delta^{i+2} 0 \supset p, \ldots, p \& q\end{array}$ & $\begin{array}{l}1, \Delta^{i+1} 0, \Delta^{i+2} 0, \ldots, \Delta 0 \supset p, \\
\Delta^{2} 0 \supset p, \ldots, \Delta^{i} 0 \supset p, \neg p, \Delta p, \\
p \vee q, p \supset q\end{array}$ \\
\hline$\ldots$ & $\ldots$ & $\ldots$ & $\ldots$ \\
\hline
\end{tabular}

Доказательство. Действительно, $f(\beta)$ есть формула (9) или (10). Поэтому $f(\beta)[\gamma]$ после распределения $\gamma$ по подформулам приобретает один из видов

$$
\begin{aligned}
& \left(C_{i+1}[\gamma] \supset C_{i}[\gamma]\right) \&\left(C_{j+1}[\gamma] \supset C_{j}[\gamma]\right) \& \ldots \&\left(C_{k+1}[\gamma] \supset C_{k}[\gamma]\right), \\
& \left(C_{i+1}[\gamma] \supset C_{i}[\gamma]\right) \&\left(C_{j+1}[\gamma] \supset C_{j}[\gamma]\right) \& \ldots \&\left(C_{k+1}[\gamma] \supset C_{k}[\gamma]\right) \& C_{l}[\gamma],
\end{aligned}
$$

где $i<j<k<l$. Теперь вспомним, что для любого $s=0,1, \ldots, l$ символом $C_{s}$ обозначена формула $\Delta^{s}(p \& \neg p)$. Поэтому результат подстановки $C_{s}[\gamma]$ не зависит от $\gamma$ и, более того,

$$
C_{s}[\gamma]=\alpha_{s} .
$$

На основании этого факта всюду в выражениях (22) и (23) заменяем $C_{s}[\gamma]$ на $\alpha_{s}$ и тем самым получаем соответственно канонические формы (4) или (5) элемента $\beta$. Тем самым утверждение (21) доказано. Лемма доказана.

Рассмотрим бесконечную последовательность одноместных предикатов

$$
\square x \leqslant \Delta 0, \square x \leqslant \Delta^{2} 0, \ldots, \square x \leqslant \Delta^{i} 0, \ldots,
$$

обозначенных ниже символами

$$
R_{1}, R_{2}, \ldots, R_{i}, \ldots
$$

Обозначим через $K_{1}, K_{2}, \ldots, K_{i}, \ldots$ классы формул, сохраняющих соответственно эти предикаты на алгебре $\mathfrak{M}^{*}$. В таблице 1 для нескольких начальных членов этой последовательности приведены примеры формул, принадлежащих классу, а также примеры формул, ему не принадлежащих.

Проверка этих примеров предоставляется читателю, однако она может быть несколько облегчена, если вместо предикатов рассматривать соответствующие им матрицы, состоящие из всех тех и только тех элементов алгебры $\mathfrak{M}^{*}$, на которых соответствующий предикат истинен. Заметим, что матрица, соответствующая предикату $R_{i}$ состоит из всех тех и только тех двоичных последовательностей $\left\langle\mu_{1}, \ldots, \mu_{i}, \ldots\right\rangle$, для которых $\mu_{i+1}=0$. Конечно, каждая из этих матриц является бесконечной, но в каждом конкретном случае достаточно ограничиться некоторой ее конечной частью. Данная формула сохраняет предикат $R(x)$ тогда и только тогда, когда соответствующая этому предикату матрица является замкнутой относительно операции, выражаемой рассматриваемой формулой. Рассматривая таблицу 1 и мысленно продолжив 
ее, легко проверить, что для любых двух классов $K_{r}$ и $K_{s}$ при условии $r<s$ имеют место соотношения

$$
\left(\Delta^{r+1} 0 \supset p\right) \in K_{r} \backslash K_{s}, \quad \Delta^{s} 0 \in K_{s} \backslash K_{r} .
$$

А этого уже достаточно, чтобы заключить, что классы $K_{1}, K_{2}, \ldots$ попарно несравнимы по включению.

Следующая лемма дает критерий принадлежности классу $K_{j}, j=1,2, \ldots$, для случая тех одноместных формул, которые при формульной реализации алгебры $\mathfrak{M}^{*}$ в логике $G L$ являются образами элементов этой алгебры.

Лемма 6. Пусть $f$ - формульная реализация алгебры $\mathfrak{M}^{*}$ в логике GL. Тогда для любого элемента $\beta$ этой алгебры и для любого $j=1,2, \ldots$ формула $f(\beta)$ принадлежит классу $K_{j}$ тогда и только тогда, когда выполнено условие

$$
\square \beta \leqslant \Delta^{j} 0 .
$$

Доказательство. В самом деле, пусть элемент $\beta$ удовлетворяет условию (24). Нужно показать, что формула $f(\beta)$ сохраняет предикат $R_{j}$ на алгебре $\mathfrak{M}^{*}$. Пусть для элемента $\gamma \in \mathfrak{M}^{*}$ истинно высказывание $R_{j}(\gamma)$. Тогда, используя $(24)$ и доказанное в лемме 5 равенство $f(\beta)[\gamma]=\beta$, находим соотношение $\square f(\beta)[\gamma] \leqslant \Delta^{j} 0$, которое означает, что $R_{j}(f(\beta)[\gamma])$. Тем самым получаем, что $f(\beta) \in K_{j}$.

Наоборот, пусть для элемента $\beta$ условие (24) не выполнено. Тогда ввиду сравнимости открытых элементов

$$
\square \beta>\Delta^{j} 0
$$

Тогда, используя опять упомянутое выше равенство (21) из леммы 5 и неравенство (25), получаем неравенство

$$
\square f(\beta)[\gamma]>\Delta^{j} 0,
$$

которое равносильно тому, что $R_{j}(f(\beta)[\gamma])$ ложно. Следовательно, формула $f(\beta)$ не принадлежит классу $K_{j}$. Лемма доказана.

Теорема 1. Пусть $L$ есть $G L$, или $L \mathfrak{M}^{*}$, или какая-то промежуточная между ними логика. Тогда существует счетное семейство предполных в логике $L$ классов формул.

Доказательство. Теорема будет следовать из следующего утверждения. Пусть логика $L$ удовлетворяет условиям

$$
G L \subseteq L \subseteq L \mathfrak{M}^{*}
$$

Тогда классы $K_{1}, K_{2}, \ldots$ формул, сохраняющих на алгебре $\mathfrak{M}^{*}$ соответственно предикаты $R_{1}, R_{2}, \ldots$, составляют счетное семейство попарно различных классов, каждый из которых предполон в $L$.

В самом деле, с помощью таблицы 1 было показано выше, что классы $K_{1}, K_{2}, \ldots$ попарно несравнимы по включению. Следовательно, ни один из этих классов не является полным в логике $L \mathfrak{M}^{*}$ и, тем самым, в логике $L$. Докажем, что при любом $j=1,2, \ldots$, класс $K_{j}$ предполон в $L$. Пусть $B\left(p_{1}, \ldots, p_{n}\right)$ - произвольная формула, не принадлежащая классу $K_{j}$. Тогда в силу определения $K_{j}$ существуют такие элементы $\beta_{1}, \ldots, \beta_{n}$ алгебры $\mathfrak{M}^{*}$, что при каждом $i=1, \ldots, n$

$$
\square \beta_{i} \leqslant \Delta^{j} 0
$$


но ложно высказывание

$$
\square B\left[\beta_{1}, \ldots, \beta_{n}\right] \leqslant \Delta^{j} 0 .
$$

В силу сравнимости открытых элементов отсюда следует сначала строгое неравенство на алгебре $\mathfrak{M}^{*}$

$$
\Delta^{j} 0<\square B\left[\beta_{1}, \ldots, \beta_{n}\right]
$$

а затем соотношение

$$
\Delta^{j+1} 0 \leqslant \square B\left[\beta_{1}, \ldots, \beta_{n}\right] .
$$

Отсюда переходим к более простому соотношению

$$
\Delta^{j+1} 0 \leqslant B\left[\beta_{1}, \ldots, \beta_{n}\right] .
$$

Поскольку $\mathfrak{M}^{*}$ является также булевой алгеброй, отсюда следует, что

$$
\left(\Delta^{j+1} 0 \supset B\left[\beta_{1}, \ldots, \beta_{n}\right]\right)=1 .
$$

Ввиду определения введенной выше формульной реализации $f$ алгебры $\mathfrak{M}^{*}$ в логике $G L$, последнее равенство приводит к соотношению

$$
\vdash f\left(\Delta^{j+1} 0 \supset B\left[\beta_{1}, \ldots, \beta_{n}\right]\right) \sim f(1) .
$$

Применяя лемму 4 к левой части этой эквиваленции, получаем, что

$$
\vdash\left(f\left(\Delta^{j+1} 0\right) \supset f\left(B\left[\beta_{1}, \ldots, \beta_{n}\right]\right)\right) \sim f(1) .
$$

Вспомнив, что $f\left(\Delta^{j+1} 0\right)$ и $f(1)$ означают формулы $\Delta^{j+1} 0$ и 1 соответственно, и применив еще раз лемму 4 , но уже к формуле $B$, находим выводимость

$$
\vdash\left(\Delta^{j+1} 0 \supset B\left[f\left(\beta_{1}\right), \ldots, f\left(\beta_{n}\right)\right]\right) \sim 1 .
$$

Левой части этой эквиваленции можно придать вид

$$
\vdash\left(\Delta^{j+1} 0 \supset \pi\right)\left[\pi / B\left[f\left(\beta_{1}\right), \ldots, f\left(\beta_{n}\right)\right]\right] \sim 1 .
$$

Заметим, что формула $\left(\Delta^{j+1} 0 \supset \pi\right)$ принадлежит классу $K_{j}$. Кроме того, поскольку элементы $\beta_{i}$ при $i=1, \ldots, n$ удовлетворяют условию $\square \beta_{i} \leqslant \Delta^{j} 0$, по лемме 6 формулы $f\left(\beta_{i}\right)$ также принадлежат классу $K_{j}$. Поэтому выводимость $(26)$ показывает, что формула 1 выразима в $G L$ через $B$ и формулы из класса $K_{j}$. Покажем, что любая формула $F$ выразима в $G L$ через систему $K_{j} \cup\{1\}$. Пусть $F$ не содержит переменную $\pi$. Тогда достаточно взять формулу $F \& \pi$, принадлежащую классу $K_{j}$, и констатировать очевидный факт, что

$$
\vdash F \sim(F \& \pi)[\pi / 1]
$$

Тем самым доказано, что система $K_{j} \cup\{B\}$ является полной в $G L$ и, тем более, в логике $L$. Сформулированное в начале доказательства утверждение, а с ним и теорема 1 , доказаны. 


\section{4. Формульные базисы любой конечной длины}

Система формул называется (функционально) независимой в логике $L$, если никакая формула этой системы невыразима в $L$ через остальные. Любую полную и независимую в логике $L$ систему формул называем формульным базисом логики $L$. Длиной формульного базиса называем число входящих в него формул. Напомним, что любой формульный базис интуиционистской логики высказываний имеет длину не более восьми. Относительно модальной логики $S 4$ и ряда ее расширений известно [11], что не существует натурального числа $k$ такого, что любой ее формульный базис имел бы длину не более $k$. Из следующей теоремы видно, что длины формульных базисов логики доказуемости $G L$ и ряда ее расширений также не ограничены никаким фиксированным числом.

Теорема 2 ([14]). Пусть $L$ есть $G L$, или $L \mathfrak{M}^{*}$, или какая-то промежуточная между ними логика доказуемости. Тогда для любого челого положительного $k$ существует формулъный базис логики $L$, имеющий мощность $k$.

Доказательство. Если $k=1$, то утверждение теоремы вытекает из того, что формула $F=\neg(p \& q) \&(\Delta q \supset \Delta r)$ является шефферовой в логике $G L$, а потому и в логике $L$. Имеет место выводимость

$$
\vdash(F[r / q] \sim \neg(p \& q))
$$

показывающая, что через $F$ выразима в $G L$ формула $\neg(p \& q)$. Ясно, что через последнюю выразимы в $G L$ формулы 0 и 1 . Поэтому на основании выводимости

$$
\vdash(F[p / 0, q / 1, r / p] \sim \Delta p)
$$

получаем, что через $F$ выразима в $G L$ формула $\Delta p$. Остается добавить, что полученная система формул

$$
\{\neg(p \& q), \Delta p\}
$$

является полной в логике $G L$. Отсюда следует утверждение теоремы при $k=1$.

Пусть теперь $k=2$. Тогда достаточно взять полную в $G L$ систему (27). Покажем, что она независима в логике $L \mathfrak{M}^{*}$ и, тем более, в $L$. Для этого достаточно заметить, что $\neg(p \& q)$ от формулы $\Delta p$ отделяется на алгебре $\mathfrak{M}^{*}$ предикатом $x \neq 0$, a $\Delta p$ от формулы $\neg(p \& q)$ отделяется на алгебре $\mathfrak{M}^{*}$ предикатом

$$
(x=y=0) \vee(x=y=1) \vee\left(x=\neg y=\alpha_{1}\right) \vee\left(\neg x=y=\alpha_{1}\right) .
$$

Далее, пусть $k \geqslant 3$. Тогда, используя знак == и читая его как означает, введем в рассмотрение три последовательности формул

$$
\begin{aligned}
A_{0}==0, \ldots, A_{i}==\Delta^{i} 0, \ldots, & & i=1,2, \ldots ; \\
\left(A_{i} \supset p\right), & & i=0,1, \ldots ; \\
C_{i}==\left(A_{i} \&\left(A_{i-1} \supset p\right)\right), & & i=1,2 \ldots
\end{aligned}
$$

Заметим для наглядности, что для любого элемента

$$
\alpha=\left\langle\mu_{1}, \mu_{2}, \ldots .\right\rangle
$$


алгебры $\mathfrak{M}^{*}$ верны равенства

$$
\begin{aligned}
A_{i}[\alpha] & =\alpha_{i}, \quad i=0,1, \ldots ; \\
\left(A_{0} \supset p\right)[\alpha] & =\langle 1,1, \ldots\rangle \\
\left(A_{i} \supset p\right)[\alpha] & =\left\langle\mu_{1}, \ldots, \mu_{i-1}, \mu_{i}, 1,1, \ldots\right\rangle, \quad i=1,2, \ldots ; \\
C_{i}[\alpha] & =\left\langle\mu_{1}, \ldots, \mu_{i-1}, 1,0,0, \ldots\right\rangle, \quad i=1,2, \ldots
\end{aligned}
$$

Имеет место следующее утверждение.

Лемма 7. При любом челом положительном $i$ формула $A_{i}$ выразима в логике $G L$ через систему формул

$$
\left\{C_{1}, \ldots, C_{i}\right\}
$$

Доказательство. Докажем при $i=1,2, \ldots$ выводимость

$$
\vdash\left(C_{i}\left[C_{i-1} \ldots\left[C_{2}\left[C_{1}\right]\right] \ldots\right] \sim A_{i}\right),
$$

показывающую, что $A_{i}$ выразима в $G L$ через систему (30). Используем индукцию по $i$. Если $i=1$ (базис индукции), то утверждение (31) сводится к частному случаю

$$
\vdash\left(C_{1} \sim A_{1}\right)
$$

вытекающему из определения формул $A_{1}$ и $C_{1}$. При $i \geqslant 2$ пусть по индуктивному предположению

$$
\vdash\left(C_{i-1}\left[\ldots\left[C_{2}\left[C_{1}\right]\right] \ldots\right] \sim A_{i-1}\right)
$$

Тогда положение (31) вытекает из очевидного факта

$$
\vdash\left(C_{i}\left[A_{i-1}\right] \sim A_{i}\right)
$$

путем замены $A_{i-1}$ подходящей эквивалентной в $G L$ формулой согласно выводимости (32). Лемма доказана.

Возвратимся к доказательству теоремы, пусть $k=3,4, \ldots$ Тогда рассмотрим систему из $k$ формул

$$
\left\{C_{1}, \ldots, C_{k-2},\left(A_{k-2} \supset p\right), T\right\}
$$

- где

$$
T==(p \& \neg q \& \Delta r) .
$$

Покажем, что она полна в логике $G L$. А именно, по лемме 7 через систему (33) выразима в $G L$ формула $A_{k-2}$. А через $A_{k-2}$ и $\left(A_{k-2} \supset p\right)$ выразима в $G L$ формула 1 на основании очевидного положения

$$
\vdash\left(\left(A_{k-2} \supset p\right)\left[A_{k-2}\right] \sim 1\right) .
$$

Легко заметить, что система $\{1, T\}$ является полной в $G L$. Значит, система (33) полна в логике $G L$. 
Докажем далее независимость системы (33) в логике $L \mathfrak{M}^{*}$. Убедимся сначала, что формула $T$ от остальных членов системы (33) отделяется на алгебре $\mathfrak{M}^{*}$ предикатом $x \neq 0$. Пусть $\alpha \neq 0$. Тогда $C_{i}[\alpha] \neq 0$ при $i=1, \ldots, k-2$, а также

$$
\left(A_{k-2} \supset p\right)[\alpha] \neq 0 \text {. }
$$

Значит, формулы $C_{i}$ и $A_{k-2} \supset p$ сохраняют предикат $x \neq 0$. Однако, когда $p=1$ и $q=1$, получаем, что $T[p / 1, q / 1]=0$, и тем самым $T$ не сохраняет на $\mathfrak{M}^{*}$ предикат $x \neq 0$.

Покажем теперь, что формула $\left(A_{k-2} \supset p\right)$ от остальных формул системы (33) отделяется на алгебре $\mathfrak{M}^{*}$ предикатом $x \neq 1$. А именно, $C_{i}[\alpha] \neq 1$ для любых элементов $\alpha \neq 1, \beta \neq 1$ и $\gamma \neq 1$ алгебры $\mathfrak{M}^{*}$ при всяком $i=1, \ldots, k-2$, а также $T[\alpha, \beta, \gamma] \neq 1$. Значит, формулы $C_{i}$ и $T$ сохраняют на $\mathfrak{M}^{*}$ предикат $x \neq 1$. Однако формула $\left(A_{k-2} \supset p\right)$ не сохраняет предикат $x \neq 1$, поскольку

$$
\left(A_{k-2} \supset p\right)\left[\alpha_{k-2}\right]=1
$$

хотя $\alpha_{k-2} \neq 1$.

Далее докажем, что при каждом $i=1, \ldots, k-2$ формула $C_{i}$ от остальных формул системы (33) отделяется на алгебре $\mathfrak{M}^{*}$ предикатом

$$
\square x \leqslant \alpha_{i-1}
$$

В самом деле, пусть элемент $\alpha$ удовлетворяет условию

$$
\square \alpha \leqslant \alpha_{i-1},
$$

означающему, что в последовательности $\alpha$ встречается нуль хотя бы на одном из первых $i$ мест. В частности, если $\alpha=\alpha_{i-1}$, то верно (35), однако имеют место равенства

$$
C_{i}\left[\alpha_{i-1}\right]=\alpha_{i} \&\left(\alpha_{i-1} \supset \alpha_{i-1}\right)=\alpha_{i},
$$

в силу которых не выполняется условие

$$
\square C_{i}\left[\alpha_{i-1}\right] \leqslant \alpha_{i-1}
$$

Значит, формула $C_{i}$ не сохраняет предикат (34). Покажем далее, что'формула $C_{j}$ при любом $j \in\{1, \ldots, k-2\} \backslash i$ сохраняет предикат (34). Пусть для $\alpha$ верно (35). Если $j<i$, то согласно положению (29) элемент $C_{j}[\alpha]$ содержит 0 по меньшей мере на $(j+1)$-м месте и тогда получаем требуемое соотношение

$$
\square C_{j}[\alpha] \leqslant \alpha_{i-1}
$$

Если же $i<j$, то, учитывая (35) и сравнимость открытых элементов, находим, что

$$
\square C_{j}[\alpha]=\square\left(\alpha_{j} \&\left(\alpha_{j-1} \supset \alpha\right)\right)=\square \alpha
$$

откуда следует, что (36) также верно.

Теперь покажем, что формула $\left(A_{k-2} \supset p\right)$ сохраняет предикат (34). Пусть для элемента $\alpha$ истинно высказывание (35). Так как $i \leqslant k-2$, в силу положения (28) у последовательностей $\alpha$ и $\left(A_{k-2} \supset p\right)[\alpha]$ первые $i$ членов совпадают. Из этого и (35) 
следует, что среди первых $i$ членов элемента $\left(A_{k-2} \supset p\right)[\alpha]$ обязательно встретится 0. Отсюда следует требуемое высказывание

$$
\square\left(A_{k-2} \supset p\right)[\alpha] \leqslant \alpha_{i-1}
$$

Наконец, остается заметить, что формула $T$ сохраняет на алгебре $\mathfrak{M}^{*}$ предикат (34). Теорема доказана.

Из теоремы 2 вытекает следующее утверждение, покрываемое теоремой 1.

Следствие 1. Пустъ $L$ естъ $G L$, или $L \mathfrak{M}^{*}$, или какая-то промежуточная между ними логика доказуемости. Тогда существует бесконечно много предполнъх в логике $L$ классов формул.

Доказательство. Допустим, что число предполных в $L$ классов формул является конечным и равно $k$. Тогда в любом множестве формул, полном в $L$, существовало бы подмножество, тоже полное а $L$, но содержащее не более $k$ элементов. Однако это противоречит теореме 2.

\section{5. Отсутствие финитной аппроксимируемости по полноте}

Согласно теореме 1 , а также следствию из теоремы 2 в любой локально нетабличной логике доказуемости существует, по меньшей мере, счетное число предполных классов формул. Поэтому для логики $G L$ и для логики $L \mathfrak{M}^{*}$, так же как и для модальной логики $S 4$, невозможно получить столь же удобный критерий полноты (по выразимости) в терминах предполных классов, как для интуиционистской логики высказываний или для модальной логики $S 5$. Дальнейшая теорема показывает еще большую сложность проблемы поиска критерия полноты в локально нетабличных логиках доказуемости.

Напомним, что та или иная логика $L$ называется финитно-аппроксимируемой, если для всякой формулы $F \notin L$ существует такое табличное расширение $L^{\prime}$ логики $L$, что $F \notin L^{\prime}$. Известно [30], что логика $G L$ является финитно-аппроксимируемой. Следовательно, она алгоритмически разрешима, то есть существует алгоритм, позволяющий по произвольной формуле $F$ распознавать, принадлежит ли она логике $G L$ (чтобы это распознать, достаточно параллельно перебирать, с одной стороны, выводы в исчислении $G L$, ища вывод $F$, и с другой стороны, конечные диагонализируемые алгебры, ища алгебру, на которой $F$ не верна).

В этой связи возникает задача, нельзя ли для данной финитно-аппроксимируемой логики $L$ и любой конечной системы формул свести вопрос о полноте последней в логике $L$ к аналогичному вопросу для табличных логик, аппроксимирующих логику $L$. Если бы для данной логики $L$ вида

$$
G L+B_{1}+B_{2}+\ldots+B_{n}
$$

эта задача решалась положительно, то существовал бы единый алгоритм распознавания полноты в $L$ для любой конечной системы формул. Ниже будет показано, что для логик $G L$ и $L \mathfrak{M}^{*}$ задача эта решается отрицательно. Поэтому проблема о существовании искомого алгоритма для них остается открытой. 
Логика $L$ называется финитно-аппроксимируемой по полноте [11], если для всякой конечной системы (формул) $\Sigma$, неполной в $L$, существует табличное расширение логики $L$, в котором система $\Sigma$ тоже неполна. В частности, финитно-аппроксимируема по полноте модальная логика $S 5$, поскольку она таблична по полноте, то есть равна по полноте (в смысле совпадения критериев) некоторой табличной логике [12].

Теорема 3. Логика GL, логика $L \mathfrak{M}^{*}$ и промежуточные между ними логики доказуемости не являются финитно-аппроксимируемыми по полноте.

Доказательство. Теорема вытекает из того, что имеет место следующее утверждение.

Система формул

$$
\{\Delta p, p \& \neg q\}
$$

не является полной в логике $L \mathfrak{M}^{*}$, однако она полна в любом табличном расширении логики $G L$.

Действительно, то, что система (37) не полна в логике $L \mathfrak{M}^{*}$, следует из того, что через нее не выразима в $L \mathfrak{M}^{*}$ формула 1 , поскольку от системы (37) она отделяется на алгебре $\mathfrak{M}^{*}$ предикатом $x \neq 1$. C другой стороны, заметим, что для любой конечной диагонализируемой алгебры $\mathfrak{A}$ существует такое целое положительное $k$, что в логике $L \mathfrak{A}$ верна эквиваленция

$$
\Delta^{k}(p \& \neg p) \sim 1
$$

показывающая, что формула 1 выразима в логике $L \mathfrak{A}$ через систему (37). Остается заметить, что система

$$
\{1\} \cup\{\Delta p, p \& \neg q\}
$$

полна в логике $L A$. Утверждение, а тем самым и теорема, доказаны.

\section{Список литературы}

1. Клини С. К., Введение в метаматематику. ИЛ, Москва, 1957.

2. Кузнецов А. В., О проблемах тождества и функциональной полноты для алгебраических систем. В кн.: Труды Третъего Всесоюзного математического съезда, m. 2. Москва, 1956, с. 145-146.

3. Кузнецов А. В. Аналоги штриха Шеффера в конструктивной логике. Докл. АН $C С C P$ (1965) 160, №2, 274-277.

4. Кузнецов А. В., О функциональной выразимости в суперинтуиционистских логиках. Математические исследования (1971) 6, №4, 75-122.

5. Кузнецов А. В., Муравицкий А. Ю., Доказуемость как модальность. В кн.: Ажтуалънъе проблемы логики и методологии науки. Наукова Думка, Киев, 1980, с. 193-230.

6. Кузнецов А. В., Раца М. Ф., Критерий функциональной полноты в классической логике предикатов первого порядка. Докл. АН СССР (1979) 249, №3, 540-544.

7. Максимова Л. Л., Континуум нормальных расширений логики доказуемости с интерполяционным свойством Крейга. Сиб. матем. ж. (1989) 30, №6, 122-131.

8. Мальцев А. И., Алгоритмы и рекурсивные функции. Наука, Москва, 1965.

9. Мальцев А. И., Итеративные алгебры Поста. Изд.-во НГУ, Новосибирск, 1976. 
10. Раца М. Ф., О функциональной полноте в интуиционистской логике высказываний. Проблемы киберн. (1982) 39, 107-150.

11. Раца М. Ф., Нетабличность логики $S 4$ по функциональной полноте. Алгебра и логиха (1982) 21, №3, 283-320.

12. Раца М. Ф., О функциональной полноте в модальной логике $S 5$. В кн.: Исследования по неклассическим логихам и формальным системам. Наука, Москва, 1983, с. 222-280.

13. Раца М. Ф., Выразимость в исчислениях высказываний. Штиинца, Кишинев, 1991.

14. Руссу А. Г., О мощности формульных базисов и отсутствии финитной аппроксимируемости по полноте в пропозициональной логике доказуемости. Buletinul A.S. a Rep. Moldova. Matematica (1992) №2(8), 6-14.

15. Фейс Р., Модальнал логика. Наука, Москва, 1974.

16. Чагров А. В. Нетабличность - предтабличность, антитабличность, ко-антитабличность. В кн.: Алгебро-логические конструхчии. Калинин, 1989, с. 105-111.

17. Чёрч А., Введение в математическую логику. ИЛ, Москва, 1960.

18. Яблонский С. В., Функциональные построения в $k$-значной логике. Труды Матем. ията им. В. А. Стеклова (1958) 51, 5-142.

19. Яблонский С. В., Введение в дискретную математиху. Наука, Москва, 1986.

20. Blok W. J., Pretabular varieties of modal algebras. Studia Logica (1980) 39, №2-3, 101-124.

21. Boolos G. S., Jeffrey R. C., Computability and logic. Cambridge Univ. Press, Cambridge, 1989.

22. Gödel K., Eine interpretation des intuitionistischen aussagen kalkulus. Ergebnisse Math. Koll. (1933), №4, 39-40.

23. Löb M., Solution of a problem of Leon Henkin. J. Symb. Logic (1955) 20, №2, 115-118.

24. Magari R., The diagonalizable algebras. Boll. Unione Matem. Italiana (1975) 12, 117-125.

25. Makinson D. C., There are infinitely many Diodorean modal functions. J. Symb. Logic (1966) 31, №3, 406-408.

26. Post E. L., Introduction to a general theory of elementary propositions. Amer. J. Math. (1921) 43, 163-185.

27. Post E. L., Two-valued iterative systems of mathematical logic. Princeton Univ. Press, Princeton, 1941.

28. Расева Е., Сикорский Р., Математика метаматематики. Наука, Москва, 1972.

29. Rosenberg I., La structure des functions de plusieure variables sur un ensemble finit. $C . R$. Acad. Sci. (1965) 260, 2817-2819.

30. Segerberg K., An essay in classical modal logic. Philosofiska Studier, Uppsala, 1971.

31. Smorinski C., Self-reference and modal logic. Springer, Berlin, 1985.

32. Solovay R. M., Provability interpretations of modal logic. Israel J. Math. (1975) 25, 287304. 